\title{
Analysis of the temporal effects on grating evolution in photopolymer
}

\author{
John V. Kelly, ${ }^{1}$ Michael R. Gleeson, ${ }^{1}$ Ciara E. Close, ${ }^{1}$ Feidhlim T. O’ Neill, ${ }^{1}$ John T. Sheridan ${ }^{1 *}$ \\ Sergi Gallego, ${ }^{2}$ Cristian Neipp ${ }^{2}$ \\ ${ }^{1}$ School of Electrical, Electronic and Mechanical Engineering, College of Engineering, Mathematics \\ and Physical Sciences, University College Dublin, Belfield, Dublin 4, Ireland. \\ ${ }^{2}$ Dpt. de Física, Ingeniería de Sistemas y Teoría de la Señal, Universidad de Alicante, Ap. 99, \\ E-03080 Alicante, Spain
}

\begin{abstract}
The nonlocal polymerization driven diffusion model is used to describe holographic grating formation in acrylamidebased photopolymer. The free radical chain polymerization process results in polymer being generated nonlocal both in space and time to the point of chain initiation. A Gaussian spatial material response function and an exponential temporal material response function are used to account for these effects.

In this paper we firstly examine the nature of the temporal evolution of grating formation for short recording periods. It is shown that in this case, temporal effects become most notable and the inclusion of the nonlocal temporal response function is shown to be necessary to accurately describe the process. In particular, brief post exposure selfamplification of the refractive index modulation is noted. This is attributed to continued chain growth for a brief period after exposure. Following this a slight decay in the grating amplitude also occurs. This we believe is due to the continued diffusion of monomer after exposure. Since the sinusoidal recording pattern generates a monomer concentration gradient during the recording process monomer diffusion occurs both during and after exposure. The evolution of the refractive index modulation is determined by the respective refractive index values of the recording material components. From independent measurements it is noted that the refractive index value of the monomer is slightly less than that of the background material. Therefore as monomer diffuses back into the dark regions, a reduction in overall refractive index modulation occurs.

Volume changes occurring within the material also affect the nature of grating evolution. To model these effects we employ a free volume concept. Due to the fact that the covalent single carbon bond in the polymer is up to $50 \%$ shorter than the van der Waals bond in the liquid monomer state, free volume is created when monomer is converted to polymer. For each bond conversion we assume a hole is generated which then collapses at some characteristic rate constant.

Incorporating each of these effects into our model, the model is then solved using a Finite-Difference TimeDomain method (FDTD). The Lorentz-Lorenz relation is used to determine the overall evolution refractive index modulation and the corresponding diffraction efficiency of the resulting grating is calculated using Rigorous Coupled Wave Analysis (RCWA). Fits are then carried out to experimental data for 1 second exposures. Good quality fits are achieved and material parameters extracted. Monomer diffusion rates are determined to be of the order of $D \sim 10^{-10}$ $\mathrm{cm}^{2} / \mathrm{s}$ and the time constant of the nonlocal material temporal response function being of the order of $\tau_{\mathrm{n}} \sim 10^{-2} \mathrm{~s}$. Material shrinkage occurring over these recording periods is also determined.
\end{abstract}

Keywords: Volume holographic gratings, holographic recording materials, polymers

\section{INTRODUCTION}

The development of accurate models to describe optical element formation in photopolymer is critical for many applications including holographic data storage ${ }^{1,2}$ and holographic optical element ${ }^{3,4,5}$ fabrication and photo-embossing. ${ }^{6}$ Many such models have been proposed. Zhao and Mouroulis ${ }^{7}$ proposed a model, which described the evolution of grating formation in photopolymer using a four harmonic expansion of the standard 1-D diffusion equation.

${ }^{*}$ Corresponding author: john.sheridan@ucd.ie; phone +353-1-716-1927; fax +353-1-283-0921

Photon Management II, edited by John T. Sheridan, Frank Wyrowski, Proc. of SPIE Vol. 6187, 618704, (2006) - 0277-786X/06/\$15 - doi: 10.1117/12.663444 
Sheridan et al. developed the nonlocal polymer driven diffusion model (NPDD) which extended the Zhao model to include a nonlocal spatial response to account for high spatial frequency cut-off - Model I. ${ }^{8}$ A square root relationship, shown to exist between the polymerization rate and the illuminating intensity, ${ }^{9,10}$ was also incorporated - Model II. ${ }^{11,12}$ Kinetics of the polymerization process have recently been examined ${ }^{13,14}$ when the chain termination mechanism is either bimolecular (two chains terminating mutually) or primary (chain terminated with a free radical) - Model III.

In this paper we first extend Model II to account for volume changes occurring in the recording material during the recording process. Karpov et al. ${ }^{15}$ and Sutherland et al. ${ }^{16}$ have examined the effect of shrinkage in photopolymer. Both assume free volume is created when monomer is converted to polymer. This is the due to the fact that the covalent single carbon bond in the polymer is up to $50 \%$ shorter than the van der Waals bond in the liquid monomer state. To model this, Sutherland et al. assumes that this results in the formation of temporary holes, which then collapse resulting in an overall reduction in the system volume. Karpov et al. allows for the diffusion of these holes throughout the medium. We assume hole collapse occurs quickly as the vacuum is filled and therefore, in this case, make the assumption that diffusion of holes is negligible. We then incorporate this behavior into the NPDD model.

The temporal response of the recording material is then examined and a material response function proposed. The model is solved using a Finite-Difference Time-Domain method (FDTD) ${ }^{17}$ and the effects of the nonlocal temporal response on refractive index modulation using the Lorentz-Lorenz relation is examined. ${ }^{18}$ These results are used to calculate diffraction efficiency using Rigorous Coupled Wave Analysis (RCWA) ${ }^{19}$ and to carry out fits to experimental data.

An inhibition process has been noted at the beginning of the recording process. This can be attributed to deactivation of excited dye molecules due to the presence of oxygen in the material. ${ }^{20}$ Models have recently been developed to describe this phenomenon. ${ }^{21}$ In this paper however we neglect this dead band region when fitting our experimental data and assume that once this inhibition is overcome the recording process in unaffected by the presence of oxygen.

\section{NONLOCAL POLYMERIZATION DRIVEN DIFFUSION MODEL, NPDD}

The generalized 1-D NPDD partial differential equation describing photopolymerization in photopolymer can be written in the form:

$$
\begin{aligned}
& \frac{\partial u(x, t)}{\partial t}=\frac{\partial}{\partial x}\left[D(x, t) \frac{\partial u(x, t)}{\partial x}\right] \\
& -\int_{-\infty}^{+\infty} \int_{0}^{t} R\left(x, x^{\prime} ; t^{\prime}\right) F\left(x^{\prime}, t^{\prime}\right) \times\left[u\left(x^{\prime}, t^{\prime}\right)\right]^{\beta} d t^{\prime} d x^{\prime} \\
& -\frac{u(x, t)}{u(x, t)+N(x, t)} \frac{\partial H(x, t)}{\partial t}
\end{aligned}
$$

where $u(x, t)$ is the free-monomer concentration, $D(x, t)$ is the monomer diffusion constant, $F(x, t)$ is the polymerization rate, $N(x, t)$ is the polymer concentration, $R\left(x, x^{\prime} ; t, t^{\prime}\right)$ is the nonlocal response function, ${ }^{8,11} H(x, t)$ is the hole concentration, and $\beta$ is a factor introduced to specify the dominant chain termination mechanism, either bimolecular $(\beta=1)$ or primary $(\beta=2){ }^{13}$ In this paper we examine the bimolecular case, $\beta=1$.

\subsection{Volume Shrinkage}

We assume that after a certain transience period the rate of hole creation will be approximately equal to the rate of hole collapse, and therefore we assume $\partial H(h, t) / \partial t$ is small and can be neglected from Eq. 1. This is a first order approximation and is valid so long as both the rate of hole formation occurring and the volume fraction of holes generated are low. The equation describing the hole formation system can be written as 


$$
\frac{\partial H(x, t)}{\partial t}=\rho \int_{-\infty}^{+\infty} \int_{0}^{t} R\left(x, x^{\prime} ; t, t^{\prime}\right) F\left(x^{\prime}, t^{\prime}\right) \times\left[u\left(x^{\prime}, t^{\prime}\right)\right]^{\beta} d t^{\prime} d x^{\prime}-k_{H} H(x, t)
$$

where $\rho$ is the fraction of free volume created for each double bond conversion and $k_{H}$ is the rate constant associated with hole collapse. Later in Section III, we present numerical results describing the effect of shrinkage on polymer

grating formation. The harmonics of hole composition can be determined where $H(x, t)=\sum_{i} H_{i}(t) \cos (i K x)$ and $H_{\mathrm{i}}$ is the $i^{\text {th }}$ harmonic component of hole concentration and $K=2 \pi / \Lambda$, where $\Lambda$ is the grating fringe spacing.

\section{$2.2 \quad$ Nonlocal Temporal Response}

The nonlocal response function represents the effect of monomer concentration at location $x^{\prime}$ and $t^{\prime}$ on the amount of material being polymerized at location $x$ and time $t$. Radical chain polymerization results in chain growth away from the point of initiation. The active tip of each chain combines with free monomer extending the polymer chain. This results in polymerization occurring nonlocal to the point of initiation as a function of both time and space. Previously ${ }^{8}$ we assumed that following a brief transient period, the spatial effect of chain growth was instantaneous (local in time or action-at-a-distance). However, where the use of short exposures is necessary, as in optical data storage, temporal effects become more significant.

It is assumed that the nonlocal response function can be broken up into the product of a spatial and a temporal response, $R\left(x, x^{\prime} ; t, t^{\prime}\right)=R\left(x, x^{\prime}\right) T\left(t, t^{\prime}\right)$. The purely temporal part of the response function takes account of the removal of monomer due to past initiations, over the time interval $0 \leq t^{\prime}<t$. In the local limit, the time response function must have the following mathematical properties:

$$
\begin{gathered}
\lim _{T_{\max } \rightarrow 0}\left\{T\left(t^{\prime}, t\right)\right\}=\delta\left(t^{\prime}-t\right), \\
\int_{-\infty}^{t} T\left(t^{\prime}, t\right) d t^{\prime}=1 .
\end{gathered}
$$

Previously it was argued that only events in the recent past, quantified using $T_{\max }$, give rise to significant nonlocal temporal effects and that at any time after $T_{\max }$ any change in monomer concentration at $x^{\prime}$ will give rise to an instantaneous change in the amount of polymerization at $x$. The time response was therefore assumed to have the property that

$$
\int_{t-T_{M A X}}^{t} T\left(t^{\prime}, t\right) d t^{\prime} \approx 1
$$

where $T_{\max }$ was defined as the maximum effective travel time between $x$ and $x{ }^{8}$ Under this assumption, the material response function reduces to a purely spatial response. This assumption is clearly questionable at times close to zero before the average number of chains reaching a point has reached a steady state and it can be assumed that only slow adiabatic variations to the steady state occur with respect to time. In this paper we no longer make this assumption but extend the nonlocal diffusion model to include both a nonlocal temporal and spatial response.

The effect of a chain initiated at time $t^{\prime}$ and position $x$ ' on the amount of polymer generated at time $t$ and position $x$ will decrease as the interval $t$ - $t$ ' increases. The biggest contribution to the removal of monomer at a point and time in space will be due to chains initiated at the same position and time. One possible temporal response function was determined to be the area normalized exponential function,

$$
T\left(t-t^{\prime}\right)=\frac{1}{\tau_{n}} \exp \left[\frac{\left(t-t^{\prime}\right)}{\tau_{n}}\right]
$$

where the time constant $\tau_{\mathrm{n}}$, determines the extent of the nonlocal temporal response. As $\tau_{\mathrm{n}}$ gets smaller the response becomes more localized and $T\left(t-t^{\prime}\right)$ approaches a delta function. The effects of introducing the nonlocal temporal response are discussed in the next section. 


\section{NUMERICAL SIMULATIONS}

Previously the nonlocal model has been solved using a two or four-harmonic expansion ${ }^{8,11}$. Recently it has been shown that for certain parameter values a more rigorous method for solving the nonlocal diffusion equation may be necessary. $\mathrm{Wu}$ and Glytsis ${ }^{17}$ applied a finite-difference time-domain (FDTD) method to solve the nonlocal diffusion equation (Model II) in its dimensionless form. Recently this method has been applied to solve the extended model, which includes the nonlocal temporal response. ${ }^{22}$ It is important to consider sampling size when using this numerical method for both stability and accuracy. For numerical stability the increment in the time domain, $\Delta t_{D}$, must satisfy the stability criterion $^{17}$

$$
\Delta t_{D} \leq \frac{1}{2} \frac{\Delta x_{D}^{2}}{R_{D}}
$$

Where $R_{D}$ is a model parameter describing the relationship between the rate of polymerization and diffusion and $\Delta x_{D}$ is the dimensionless time step. In most cases we choose $\Delta t_{D}=0.4\left(\Delta x_{D}{ }^{2} / R_{D}\right)$, which is consistent with the Wu and Glytsis analysis $^{17}$. However the diffusion model now includes a time integral. In evaluating this integral, using the trapezoidal rule, the size of $\Delta t_{D}$ is critical to the numerical accuracy of the result. To estimate a suitable value of $\Delta t_{D}$, we examine the truncation error associated with the trapezoidal rule. Using the Taylor series it can be shown that the modulus of the truncation error, $|e|$, for the integral, $\int_{0}^{t_{D}{ }^{\prime}} T\left(t_{D}, t_{D}{ }^{\prime}\right) d t_{D}$, is given by ${ }^{23}$

$$
|e| \leq \frac{1}{12} \Delta t_{D}^{2}\left|T^{\prime \prime}(\tau)\right| \times t_{D}
$$

where $\left|T^{\prime \prime}(\tau)\right|=\max \left\{\left|T\left(x, t_{D}{ }^{\prime}\right)\right| 0 \leq x \leq t_{D}{ }^{\prime}\right\}$.

By choosing a suitable value for the maximum allowable truncation error in our system, we can then estimate an appropriate value for $\Delta t_{D}$. The truncation error used was $|e|=0.005$.

The NPDD model is solved for the harmonic components of monomer, polymer and hole concentration during grating formation. Previously, as discussed in Section 2, it has been assumed that rates of polymerization respond instantaneously to changes in light intensity, i.e. there is no temporal response. Therefore if illumination is stopped during grating formation, polymerization stops instantaneously. Based on the above analysis and assuming that all the monomer has not been consumed completely by the end of illumination, we would expect initiated chains to continue growing after exposure before terminating some short time after. Colvin et al. ${ }^{24}$ examined the harmonic evolution of polymer after exposure. However any subsequent change in the harmonic amplitude was attributed solely to monomer diffusion and the effects of "dark reactions", i.e. continued polymer growth after illumination, were ignored. In this section we examine the effects of the inclusion of a nonlocal temporal response on harmonic evolution post exposure while considerable unpolymerized monomer still remains available in the material and we also examine the influence of polymer shrinkage.

\subsection{Effect of temporal response}

Figure 1 shows the effect of dimensionless spatial and temporal nonlocal parameters, $\sigma_{n D}$ and $\tau_{n D}$ respectively, on the polymer and monomer first harmonic evolution when recording is stopped after $t_{\mathrm{D}}$ (dimensionless time) $=0.1$ and where $\beta=1$. 


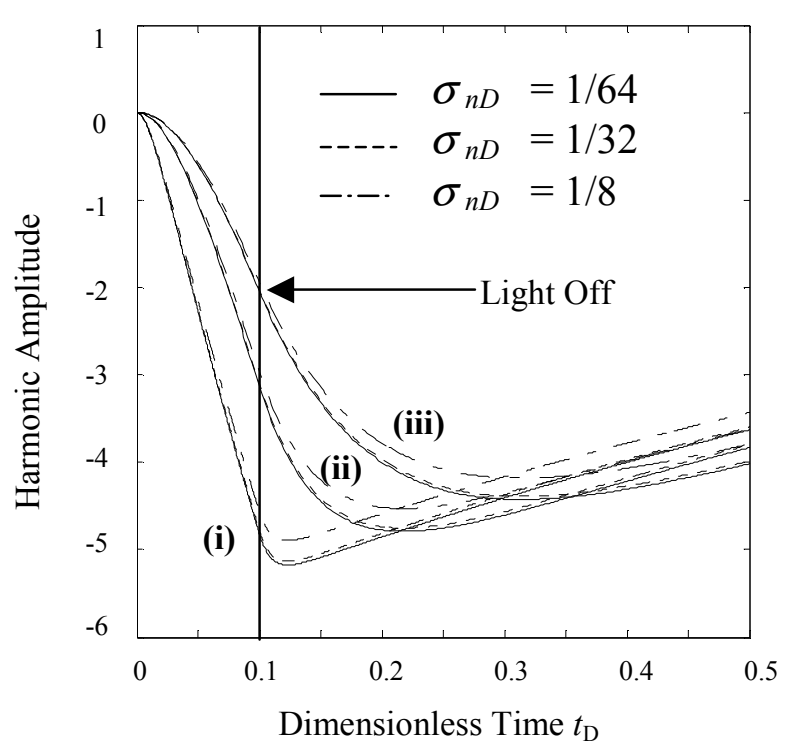

(a)

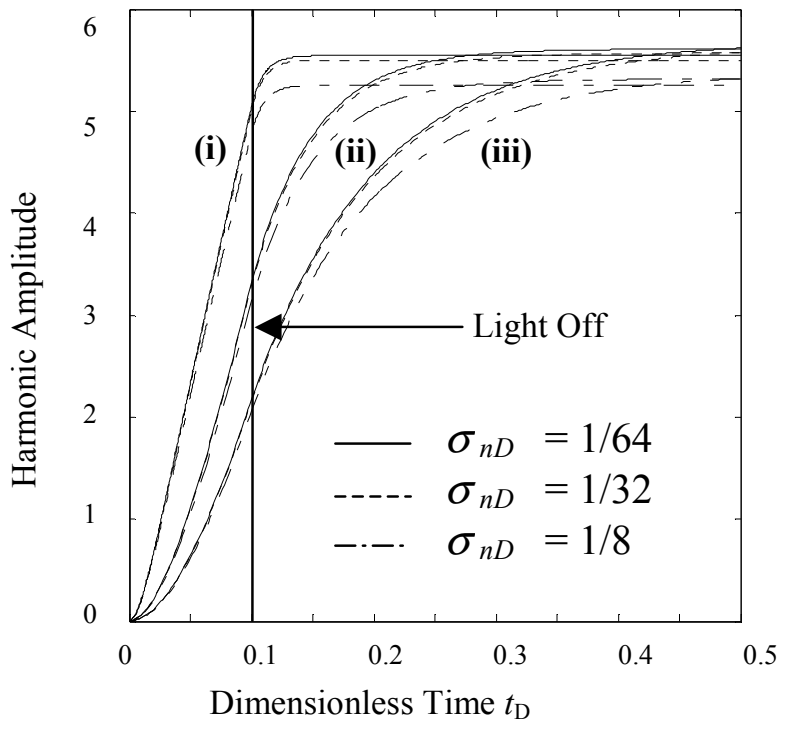

(b)

Figure 1. First harmonic coefficient of (a) monomer concentration and (b) polymer concentration for values of $\sigma_{n D}=(1 / 64,1 / 32$, $1 / 8$ ) and $\tau_{n D}=$ (i) 0.01 , (ii) 0.05 , (iii) 0.1 where $R_{D}=1$ and $\beta=1$.

For small values of $\tau_{n D}$ we see that there is little increase in polymer harmonic amplitude after exposure has stopped.

However, as the value of $\tau_{n D}$ increases the amplitude of the polymer harmonic continues to increase significantly before saturating. Similarly the monomer harmonic amplitude decreases as the monomer diffuses back into the depleted regions of the grating layer. Assuming chain termination is not instantaneous once exposure has stopped, and based on the above simulation, we see continued polymerization for a short period after exposure.

\subsection{Effect of hole formation}

The rate at which holes are generated and collapse will depend on the characteristics of the material system being examined. Holes are generated as polymer is formed during the recording process. When recording stops the holes continue to collapse. For longer exposures the hole concentration will decrease before reaching a constant value, during the process. In this case however the rate of collapse never exceeds the rate of generation during the brief recording period and therefore never reaches a steady state value. However as discussed in Section 2, given the slow rate of hole generation and collapse, and the low concentration of holes generated in this period, our first order approximation appears valid. In the next section we examine the influence of hole formation on the refractive index modulation.

\section{REFRACTIVE INDEX MODULATION EVOLUTION}

In this section we show how the refractive index evolution is determined based on the Lorentz -Lorenz relation and the refractive index values of the individual photopolymer material components.

\subsection{Refractive index modulation}

Following the analysis of Aubrecht et al., ${ }^{1822}$ the refractive index modulation can be written as

$$
n_{1}=\frac{\left(n_{\mathrm{dark}}^{2}+2\right)^{2}}{6 n_{\mathrm{dark}}}\left[\phi_{1}^{(m)}\left(\frac{n_{m}{ }^{2}-1}{n_{m}{ }^{2}+2}-\frac{n_{b}{ }^{2}-1}{n_{b}{ }^{2}+2}\right)+\phi_{1}^{(p)}\left(\frac{n_{p}{ }^{2}-1}{n_{p}{ }^{2}+2}-\frac{n_{b}{ }^{2}-1}{n_{b}{ }^{2}+2}\right)-\phi_{1}^{(H)}\left(\frac{n_{b}{ }^{2}-1}{n_{b}{ }^{2}+2}\right)\right]
$$


where $n_{\text {dark }}$ is the average refractive index of the material before exposure. $\phi_{1}{ }^{(\mathrm{m})}, \phi_{1}{ }^{(\mathrm{p})}$ and $\phi_{1}{ }^{(\mathrm{H})}$ are the first harmonic components of monomer, polymer and hole concentration respectively and $n_{m}, n_{p}, n_{b}$ are the refractive index values of monmer background material and polymer respectively. The refractive index values of $n_{m}$ and $n_{b}$ were independently measured using a metricon prism coupler where $n_{m} \approx 1.47$ and $n_{b} \approx 1.49$. We can then estimate $n_{\text {dark }}$ using Eq. 7 with the initial volume fraction of monomer, $\phi_{1}{ }^{(\mathrm{m})}$, taken to be 0.13 . This gives $n_{\text {dark }} \approx 1.49$. Using Eq. 7 and the harmonic values determined in Section 3 we now examine the evolution of the refractive index modulation as a function of time.

The values estimated for the refractive index of the monomer and background indicate $n_{\mathrm{b}}>n_{\mathrm{m}}$. Therefore as the monomer diffuses back into the polymerized regions post-exposure, the refractive index in these regions is reduced and hence the refractive index modulation decreases. Hole generation will also have an effect. The initial growth in refractive index modulation is reduced due to the presence of holes, having a refractive index value of $n_{\mathrm{H}}=1$. Postexposure however two opposing processes exist. Collapsing holes will cause the refractive index modulation to increase while the diffusion of monomer will continue to cause a reduction in the refractive index modulation. In each case, as the holes collapse, the value of the refractive index modulation tends towards the same saturation value. In the next section, we examine experimental results.

\section{EXPERIMENTAL RESULTS}

The photopolymer solution is deposited on glass slides and allowed to dry for 48 hours. The resulting plates have a thickness of $90 \pm 5 \mu \mathrm{m}$, which can be measured directly using a micrometer screw gauge. Unslanted transmission holographic gratings were recorded with a spatial frequency of 1000 lines $/ \mathrm{mm}$. Recording was carried out using a $532 \mathrm{~nm}$ solid-state laser with a recording intensity of $2 \mathrm{~mW} / \mathrm{cm}^{2}$. A $633 \mathrm{~nm} \mathrm{HeNe} \mathrm{laser} \mathrm{was} \mathrm{used} \mathrm{to} \mathrm{monitor} \mathrm{the} \mathrm{first}$ order on-Bragg diffraction efficiency. Short exposure experiments were carried out, each for a different exposure time. Exposure time was controlled using a shutter, which closes after the required recording time has elapsed. We continue to monitor the diffraction efficiency for a period after this point.

\section{FITS TO EXPERIMENTAL DATA}

In a recent publication fits to this experimental data have been presented for short exposures using the nonlocal model, ${ }^{22}$ assuming shrinkage to be negligible. Good fits were achieved with parameter fit values for refractive index corresponding closely to those determined independently.

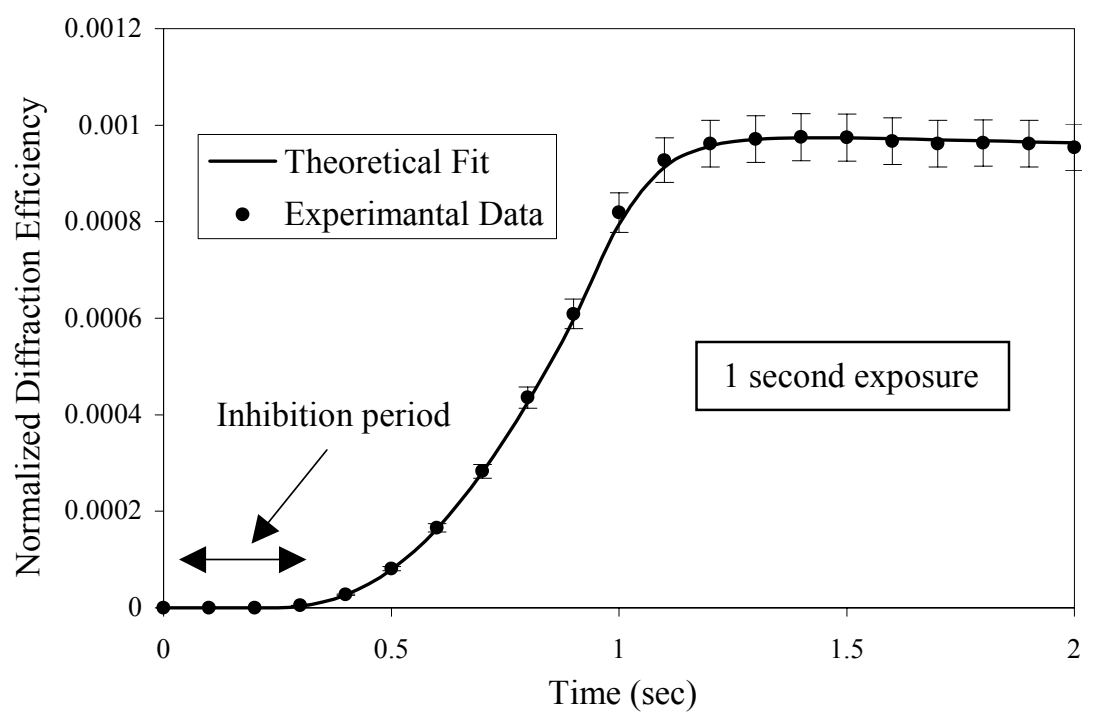

Figure 2. Theoretical fit to experimental data using the best fit parameters given in Table 1 for a 1 second exposure. 
We now fit the data using the same procedure described in Ref. 22, for the new extended model. Examining the experimental data it is evident that the evolution of the profile for each curve is not exactly identical to the previous exposure. However up to one second the repeatability is good. Therefore we apply our fitting procedure to the onesecond-exposure data set. The results are shown in figure 2 and the best fit parameter values are shown in Table 1.

\begin{tabular}{ccccccccccc}
\hline $\begin{array}{c}\text { Exposure } \\
\text { Time } \\
(\mathbf{s e c})\end{array}$ & $n_{p .}$ & $n_{b}$ & $n_{\mathrm{m}}$ & $\tau_{n}(\mathrm{sec})$ & $\begin{array}{c}\sqrt{\sigma^{\prime}} \\
(\mathbf{n m})\end{array}$ & $\boldsymbol{R}$ & $\begin{array}{c}\boldsymbol{D}\left(\mathrm{cm}^{2} / \mathbf{s}\right) \\
\left(\mathbf{x ~ 1 0} \mathbf{1 0}^{-10}\right)\end{array}$ & $\boldsymbol{k}_{\mathrm{H}}\left(\mathbf{s}^{-1}\right)$ & $\rho$ & $\begin{array}{c}\text { MSE } \\
\left(\mathbf{x ~ 1 0} \mathbf{1 0}^{-11}\right)\end{array}$ \\
\hline 1 & 1.511 & 1.495 & 1.493 & 0.09 & 60 & 8 & 2 & 0.7 & 0.0005 & 6.4 \\
\hline
\end{tabular}

Table 1. Best fit parameters obtained for a 1 second exposure.

A good fit is achieved with a Mean Square Error (MSE) of $6.4 \times 10^{-11}$. However the volume of holes relative to the volume of polymer generated over this period is small $(\rho=0.005)$ and the results would appear to support the assumption that for short exposures little or no shrinkage occurs and therefore volume changes do not influence significantly the nature of grating formation examined here. Hole decay, post-exposure may also cause some initial grating amplification. However once again we expect this effect to be small due to the slow hole decay rate and the low concentration of holes generated.

\section{DISCUSSION AND CONCLUSION}

In this paper we have investigated the inclusion of a nonlocal temporal response in the NPDD model. Numerical results have shown that as the extent of the nonlocal temporal response changes, the nature of the evolution of the polymer harmonics at the initial stages of exposure also changes. Examining the effect of the inclusion of this response when modelling grating evolution after exposure has stopped, and based on the assumption that chain termination is not instantaneous, we have carried out numerical simulations, which predict continued polymerization for a period after exposure. We have also extended the nonlocal model to account for material shrinkage, which occurs during the polymerization process and examined the nature of hole formation and collapse. We have estimated the refractive index of the main components of the photopolymer recording material. Based on these results and the results of the nonlocal diffusion model and using the Lorentz-Lorenz relation we have examined the evolution of the refractive index modulation during short exposures and also immediately post-illumination. It has been shown that with the inclusion of a nonlocal temporal response, the index modulation continues to increase after illumination and then decreases as the amplitude of the monomer grating decreases due to monomer diffusion. Initial results would appear to indicate that for short exposure the influence of volume changes in the material is negligible.

We note that this is a first order examination of hole formation in photopolymer and is valid under the assumptions made in Section II. Initial results would appear to indicate that it provides a good approximation to the effects of shrinkage on grating formation. However a more rigorous solution to the NPDD model presented may be necessary to fully describe this behavior. We also note that previously ${ }^{22}$ superior fits to experimental data were achieved using the primary termination model $(\beta=2)$ when compared to the bimolecular case $(\beta=1)$. Further work is necessary to compare these two cases using the model presented here.

\section{ACKNOWLEDGEMENTS}

We acknowledge the support of Enterprise Ireland and Science Foundation Ireland through the through the Research Innovation Fund, the Basic Research Program and the Research Frontiers Program and of the Irish Research Council for Science, Engineering and Technology. One of the authors (JVK) holds an Irish Canadian University Foundation (ICUF) scholarship. 


\section{REFERENCES}

1 H. J. Coufal, D. Psaltis, G. T. Sincerbox (eds), Holographic Data Storage, Springer Series in Optical Sciences Series, Springer, Berlin, (2000).

2 J. T. Sheridan, M. R. Gleeson, J. V. Kelly, F. T. O’Neill, "Nonlocal polymerisation driven diffusion model based examination of the scaling law for holographic data storage," Opt. Lett. 30, No. 3, 239-241, (2005).

3 H. Kobolla, J. T. Sheridan, E. Gluch, J. Schmidt, R. Völkel, J. Schwider, N. Streibl, "Holographic 2-D mixed polarisation deflection elements", J. Mod. Opt. 40, No. 4, 613-624 (1993).

4 H. Kobolla, J. Schmidt, J. T. Sheridan, N. Streibl, R. Völkel, "Holographic optical beamsplitters in dichromated gelatin", J. Mod. Opt. 39, No. 4, 881-887 (1992).

5 J. Schmidt, R. Völkel, W. Stork, J. T. Sheridan, J. Schwider, N. Streibl, F. Durst, "Diffractive beamsplitter for laser doppler velocimetry", Opt. Lett. 17, 1240-1242 (1992).

6 F. T. O’Neill, A. J. Carr, S. M. Daniels, M. R. Gleeson, J. V. Kelly, J. R. Lawrence, J. T. Sheridan, "Refractive elements produced in photopolymer layers," J. Mat. Sci. 40, 4129-4132 (2005).

7 G. Zhao, P. Mouroulis, "Diffusion model of hologram formation in dry photopolymer materials," J. Mod. Opt. 41, 1929-1939 (1994).

8 J. T. Sheridan, J. R. Lawrence, "Non-local response diffusion model of holographic recording in photopolymer," J. Opt. Soc. Am. A 17, 1108-1114 (2000).

9 J. H. Kwon, H. C. Chang, K. C. Woo, "Analysis of temporal behavior of beams diffracted by volume gratings formed in photopolymers," J. Opt. Soc. Am. B 16, 1651-1657 (1999).

10 G. Odian, Principles of Polymerization (Wiley, New York, 1991).

11 J. R. Lawrence, F. T. O’Neill, J. T. Sheridan, "Adjusted intensity nonlocal diffusion model of photopolymer grating formation,” J. Opt. Soc. Am. B 19, 621-624 (2002).

12 Justin R. Lawrence, Feidhlim T O’Neill, John T. Sheridan, "Photopolymer holographic recording material parameter estimation using a nonlocal diffusion based model," J. Appl. Phys. 90, 3142-3148 (2001).

13 J. V. Kelly, F. T. O’Neill, J. T. Sheridan, "Holographic photopolymer materials: non-local polymerisation driven diffusion under non-ideal kinetic conditions," J. Opt. Soc. Am. B 22, 407-416 (2005).

14 S. Blaya, L. Carretero, R. F. Madrigal, M. Ulibarrena, P. Acebal, A. Fimia, "Photopolymerization model for holographic gratings formation in photopolymers," Appl. Phys. B 77, 639-662 (2003).

15 H. M. Karpov, V. V. Obukhovsky, T. N. Smirnova, "Generalized model of holographic recording in photopolymer materials," Semiconductor Physics, Quantum Electronics and Optoelectronics 2, 66-70 (1999).

16 R. L. Sutherland, V. P. Tondiglia, L. V. Natarajan, and T. J. Bunning, "Phenomenological model of anisotropic volume hologram formation in liquid- crystal-photopolymer mixtures," J. Appl. Phys. 96, 951-965 (2004).

17 S. Wu, E. N. Glytsis, "Holographic grating formation in photopolymers: analysis and experimental results based on a nonlocal diffusion model and rigorous coupled-wave analysis," J. Opt. Soc. Am. B 20, 1177-1188 (2003).

18 I. Aubrecht, M. Miller, I. Koudela, "Recording of holographic gratings in photopolymers: theoretical modelling and real-time monitoring of grating growth," J. Mod. Opt. 45, 1465-1477 (1998).

19 M. G. Moharam, T.K. Gaylord, "Rigorous Coupled-Wave analysis of planar-grating diffraction,” J. Opt. Soc. Am. 71, 811-818 (1981).

20 A. V. Galstyan, R. S. Hakobyan, S. H, T.Galstian, "Study of the inhibition period prior to the holographic grating formation in liquid crystal photopolymerizable materials" http://www.elc.org/Documents/T._V_Galstian_2004_05_05_11_13_17.pdf

${ }^{21}$ M. R. Gleeson, J. V. Kelly, C. E. Close, F. T. O’Neill, J. T. Sheridan, "The Impact of Inhibition Processes during Grating Formation in Photopolymer Materials", in Photonic Engineering, R. F. O'Dowd, ed., Proc. SPIE 5827, 232-243, (2005).

22 J. V. Kelly, M. R. Gleeson, F. T. O’Neill, J. T. Sheridan, C. Neipp, S. Gallego, M. Ortuno, "Temporal analysis of grating formation in photopolymer using the nonlocal polymerization-driven diffusion model," Opt. Exp. 13, 6990-7004 (2005).

23 C. Dixon, Numerical Analysis, Blackie, (Glasgow and London, 1982).

24 V. L. Colvin, R. G. Larson, A. L. Harris, M. L. Schilling, "Quantitative model of volume hologram formation in photopolymers," J. Appl. Phys. 81, 5913-5923 (1997). 